\title{
INVESTIGATION OF ABNORMAL GRAIN GROWTH IN A FRICTION STIR WELDED AND SPIN-FORMED AL-LI ALLOY 2195 CREW MODULE
}

\author{
Wesley A. Tayon, Marcia S. Domack, Eric K. Hoffman, Stephen J. Hales \\ NASA Langley Research Center, Hampton, VA 23681, USA
}

Keywords: Abnormal grain growth, Al-Li alloy, friction stir weld, spin-forming

\begin{abstract}
In order to improve manufacturing efficiency and reduce structural mass and costs in the production of launch vehicle structures, NASA is pursuing a wide-range of innovative, near-net shape manufacturing technologies. A technology that combines friction stir welding (FSW) and spin-forming has been applied to manufacture a single-piece crew module using AluminumLithium (AL-Li) Alloy 2195. Plate size limitations for Al-Li alloy 2195 require that two plates be FSW together to produce a spin-forming blank of sufficient size to form the crew module. Subsequent forming of the FSW results in abnormal grain growth (AGG) within the weld region upon solution heat treatment (SHT), which detrimentally impacts strength, ductility, and fracture toughness. The current study seeks to identify microstructural factors that contribute to the development of AGG. Electron backscatter diffraction (EBSD) was used to correlate driving forces for AGG, such as stored energy, texture, and grain size distributions, with the propensity for AGG. Additionally, developmental annealing treatments prior to SHT are examined to reduce or eliminate the occurrence of AGG by promoting continuous, or uniform, grain growth.
\end{abstract}

\section{Introduction}

The current NASA crew module design is a multi-piece, friction stir welded construction that relies on traditional manufacturing methods and significant assembly labor. Advanced manufacturing methods are being explored to reduce structural weight, simplify and lower the cost of fabrication and increase design margins. A novel crew module concept fabricated by spin-forming Al-Li alloy 2195 plate combines multiple structural elements into a single-piece, eliminating twelve longitudinal and three circumferential welds, and achieving approximately $10 \%$ reduction in vehicle weight. In order to produce a sufficiently large spin forming blank, two commercial scale 2195 plates must be joined together by FSW.

The necessity to FSW two plates of Al-Li alloy 2195 prior to spin-forming creates metallurgical challenges, such as AGG, which must be addressed. Generally, a microstructure produced via FSW consists of a fine-grained, dynamically recrystallized microstructure. However, it has frequently been demonstrated that such microstructures are unstable during exposure to SHT temperatures during subsequent heat treatment $[1,2]$. This leads to AGG in the weld nugget, transforming the as-welded, fine-grained microstructure into one consisting of just a few grains with dimensions of several millimeters. Prior research indicated that the extent of AGG was magnified with an increasing level of spin-forming deformation [3]. 
AGG within the FSW poses the greatest risk in terms of meeting mechanical property specifications. In a prior study [4], tensile tests of FSW regions with AGG exhibited low ductility $(<1 \%)$ and failed in the AGG regions. However, mechanical properties of FSW Al-Li alloys tend to be comparable to base plate material, if the fine-grained microstructure can be retained. Hence, a thorough examination of the microstructure in various intermediate processing conditions is warranted to develop thermal processing strategies to eliminate AGG, in order to successfully produce a FSW and spin-formed structure with optimized mechanical properties. Exploratory IATs (intermediate annealing treatments) are examined as means to promote uniform grain growth in order to reduce differentials in grain size between isolated and matrix grains. Based on the Humphreys' model $[5,6]$, promoting continuous or uniform grain growth may suppress AGG upon SHT. The IAT is applied after spin-forming and before SHT. Prior work has shown that an IAT can significantly reduce the extent of AGG [3].

AGG is characterized by the rapid, preferential growth of isolated grains that consume the smaller matrix grains and grow to several millimeters in length. AGG results from microstructural instability, typically associated with fine-grained, heavily deformed microstructures. Humphreys and others have investigated the issue of microstructural stability and AGG in various Al alloys [5-12]. Several critical factors have been identified as driving forces for AGG: grain size and distribution; stored energy (deformation) gradients; grain orientation (texture); and grain boundary misorientation. Additionally, several retarding forces were identified: Zener drag linked to insoluble dispersoid particles and soluble precipitate; and solute drag related to matrix solute content. Specifically, AGG is observed for isolated grains that possess that the follow characteristics: larger grain size; lower stored energy; increased boundary mobility (high-angle grain boundaries and certain coincident site lattice (CSL) boundary types); and the absence of effective boundary pinning by insoluble dispersoids, soluble precipitates, and/or matrix solute content compared to the surrounding matrix grains.

In contrast to primary recrystallization driven by strain energy, AGG (sometimes referred to as secondary recrystallization) is driven by interfacial boundary energy, i.e. the growth of existing grains. Whether normal (continuous) or abnormal (discontinuous) grain growth occurs depends on the homogeneity of grain boundary mobility within the incumbent microstructure. It is evident that the driving (and retarding) forces for AGG are so diverse that a unified model has not been forthcoming [13]. The common feature of most theories is that the net driving force is manifested as grain boundary mobility distributions, i.e. the more inhomogeneous, the more likely that AGG will occur. Of particular significance to this research is the recent work reported by Chen et al., which found a correlation between AGG and the population of low-angle grain boundaries (LAGBs) within an Al 5083 alloy FSW, i.e.. AGG occurred in regions that had >18\% LAGBs [1].

In this study, confidence weld plates produced with the same FSW procedures and parameters as the spin-forming blank were hot rolled $25 \%$ and exposed to various IAT conditions. Samples were then given a SHT to determine the optimum IAT parameters based on the extent of AGG exhibited. EBSD was used to evaluate the microstructural changes promoted by the IAT, following post-FSW deformation via hot rolling. The objective of this study was to quantify microstructural factors relative to AGG susceptibility. The most successful IAT was examined in terms of identifying the microstructural changes that served to suppress AGG. The effects of 
soluble precipitates, matrix solute content, and dispersoid particles were reserved for future efforts.

\section{Experimental Procedure}

Two 40 mm-thick Al-Li alloy 2195-T3M4 plates were FSWed together using a double-pass weld (plates were welded from the top and bottom of the plate) along the rolling direction. For experimental purposes, confidence weld material was produced to examine the effect of deformation and IATs on the occurrence of AGG within the FSW to ultimately guide processing of the spin-forming blank. Both the confidence weld plate and spin-forming blank were given a post-weld anneal (PWA). Confidence weld material was hot rolled (HR) near spin-forming temperatures to a $25 \%$ thickness reduction to generate widespread AGG after SHT. The $25 \%$ level of deformation represented the maximum estimated forming strain by the vendor for complex components. Meanwhile, the spin-formed dome in this study does not encounter forming strains of that magnitude. However, if the IAT approach is successful for material with $25 \%$ deformation, its validity will be established for application to more complex shapes for future structures. A piece of the $25 \%$ hot rolled material was SHTed to develop a benchmark for AGG. The remaining hot rolled material was consumed to experiment with various IATs designed to suppress AGG.

Samples in the As FSW, FSW+ 25\% HR, and FSW+ 25\% HR + IAT conditions were extracted from the confidence weld material and examined using EBSD perpendicular to the weld direction in the S-T plane. For comparison to the HR confidence plate, a piece from the as spinformed dome was also evaluated with EBSD. Texture was examined with inverse pole figure (IPF) maps referenced to the thickness direction (S) of the plate. Grain sizes were computed from EBSD data based on grain area and diameter. The grain boundary misorientation angle distribution (GBMAD) was assessed in each processing condition and compared to prior data to analyze variations in grain boundary mobility.

\section{Results and Discussion}

Optical micrographs of confidence weld material in the 'As FSW', after 25\% HR + SHT, and after 25\% HR + IAT + SHT conditions are shown in Figure 1 alongside a sample from the spinformed dome after SHT. The As FSW microstructure (Figure 1A) consists of a fine-grain structure, which is largely stable upon subsequent SHT. The introduction of deformation by HR destabilizes the weld nugget region and leads to AGG upon SHT throughout nearly the entire weld region (Figure 1B). Application of an IAT significantly reduces the extent of AGG by $60 \%$, particularly at the quarter-thickness, or $\mathrm{t} / 4$, region (Figure $1 \mathrm{C}$ ). The spin-formed condition

only exhibits a small amount of AGG in the overlap region (Figure 1D). A similar level of AGG was found in the As FSW condition after PWA, indicating an increased susceptibility to AGG at the overlap region. The elimination of AGG at the overlap region likely will not be achieved through thermal treatments alone, and appears to require optimization of the weld parameters or tooling. The near absence of AGG in the spin-formed dome is attributed to lower forming strains. The thickness reduction after spin-forming was $\sim 5 \%$ compared to the $25 \%$ reduction via hot rolling for the confidence weld plate. 


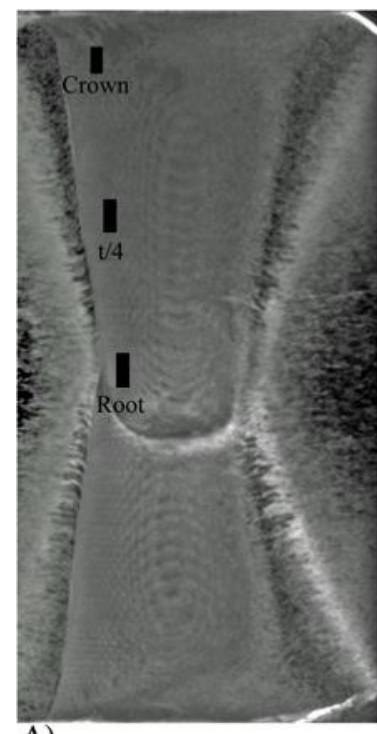

A)

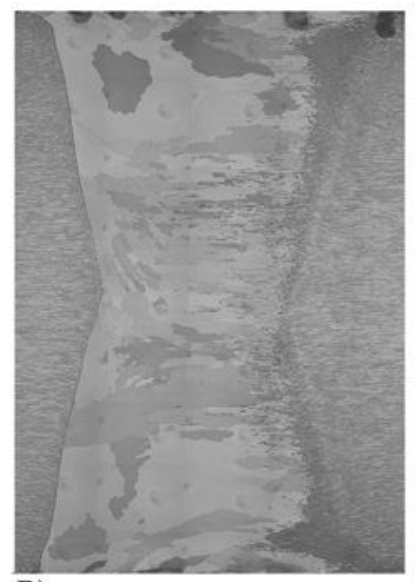

B)

$2 \overrightarrow{\mathrm{mm}}$
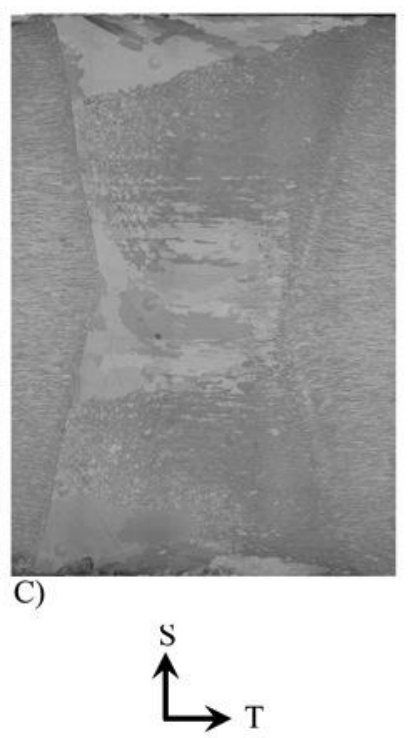

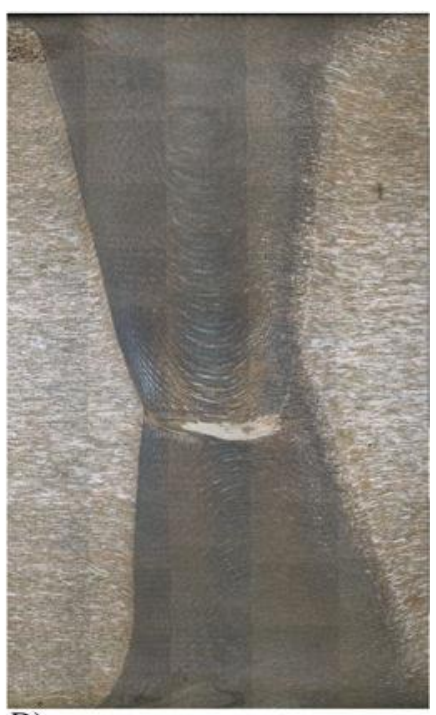

D)

Figure 1 Optical micrographs for the A) As FSW-only; B) After 25\% HR + SHT; C) After 25\% HR + IAT + SHT; and D) Spin-Formed + SHT conditions.

EBSD data was collected at the crown, $t / 4$, and root regions of the As FSW condition indicated in Figure 1A by the black boxes. The IPF maps for these regions reveal significant gradients in both grain size and grain orientation throughout the weld as seen in Figure 2. Average grain sizes for each reach are listed in Table 1. The largest grains sizes were measured at the crown region. No preferred texture is found in the crown region. At $t / 4$, the grain size decreased and a preferred shear texture emerges (dominant $\langle 111\rangle$ texture). Further grain size reduction was evident at the root region with a slightly stronger shear texture than $t / 4$. The differences in grain size and orientation (texture) indicated variability inherent in the As FSW microstructure. This leads to variability in the driving forces for AGG.

Table 1 Average grain area in $\mu \mathrm{m}^{2}$ for each of the regions indicated in the As FSW condition. Average grain diameters in $\mu \mathrm{m}$ are noted in parentheses.

\begin{tabular}{|c|c|c|}
\hline Crown & t/4 & Root \\
\hline $69.0(9.4)$ & $41.6(7.3)$ & $18.2(4.8)$ \\
\hline
\end{tabular}

The GBMAD for the crown, $t / 4$, and root regions of the As FSW condition are plotted in Figure 3. In relation to AGG, the GBMAD has been empirically correlated with grain growth and mobility [14]. Typically, grain boundaries are categorized as either LAGBs or HAGBs, with a threshold selected at $15^{\circ}$. Generally, LAGBs are less mobile than high-angle grain boundaries (HAGBs). As the number of LAGBs increases, the potential for AGG increases. Comparing the three regions of the weld, the crown contains the highest number of HAGBs and only $8.5 \%$ LAGBs, similar to the theoretical random distribution [15]. Progression through the thickness of the weld to the root region is accompanied by an increase in the percentage of LAGBs. Typically, the root is associated with lower heat input and higher deformation (stored energy) compared to other regions of the weld [3]. The root region displays a bi-modal distribution of LAGBs and HAGBS, with $\sim 25 \%$ LAGBs. This region of the FSW is the most susceptible region 
to AGG upon thermal exposure, which is attributed to highly anisotropic grain boundary mobility. This observation is consistent with a documented correlation between AGG and the percentage of LAGBs within an FSW in a study by Chen [1]. All regions exhibiting AGG had a percentage of LAGBs exceeding $18 \%$. These regions were identified as regions of high-strain and lower temperature during FSW. Regions with less that $18 \%$ LAGBs did not exhibit AGG.
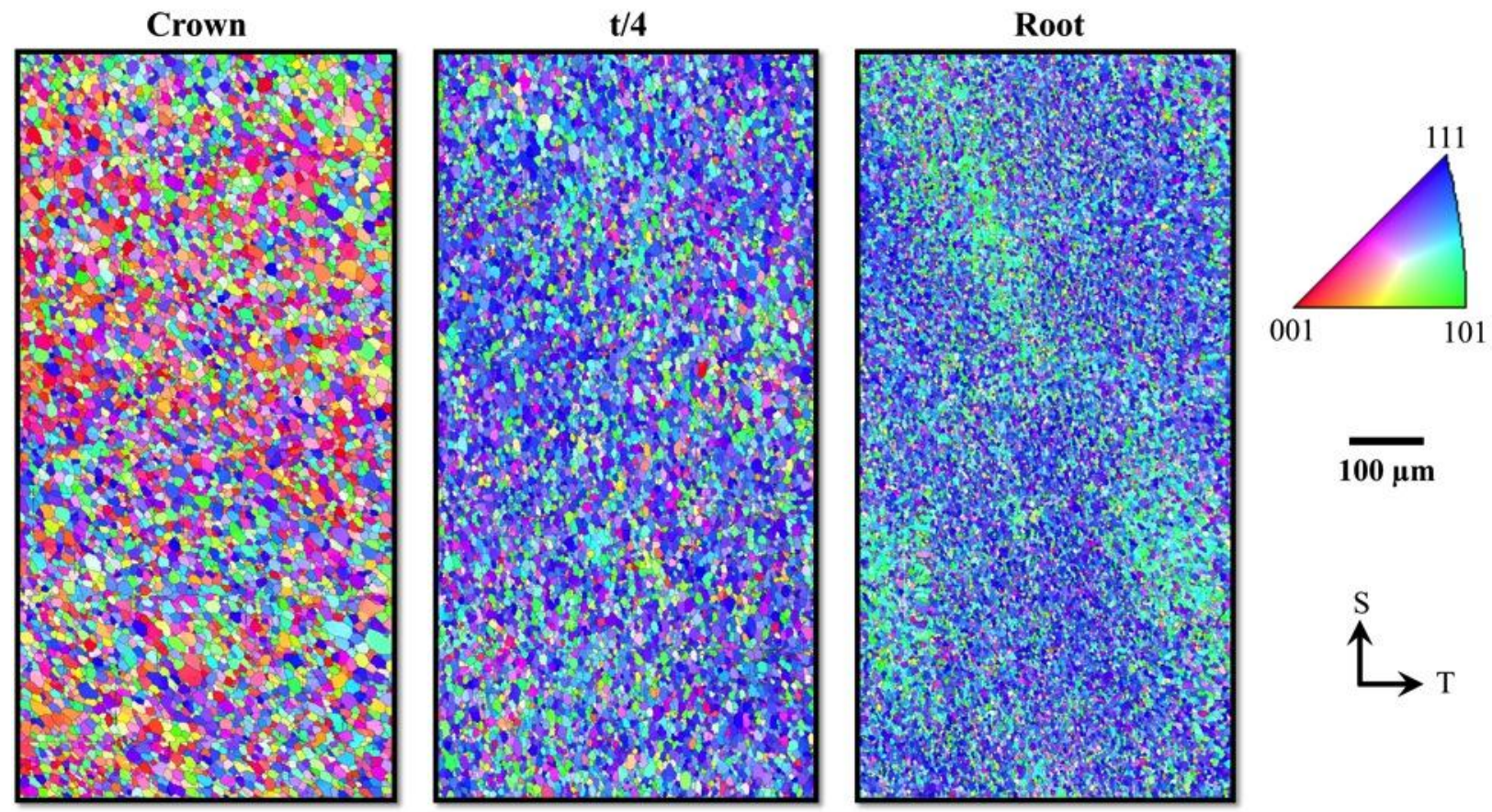

Figure 2 IPF maps for the crown, t/4 and root locations in the As FSW condition.

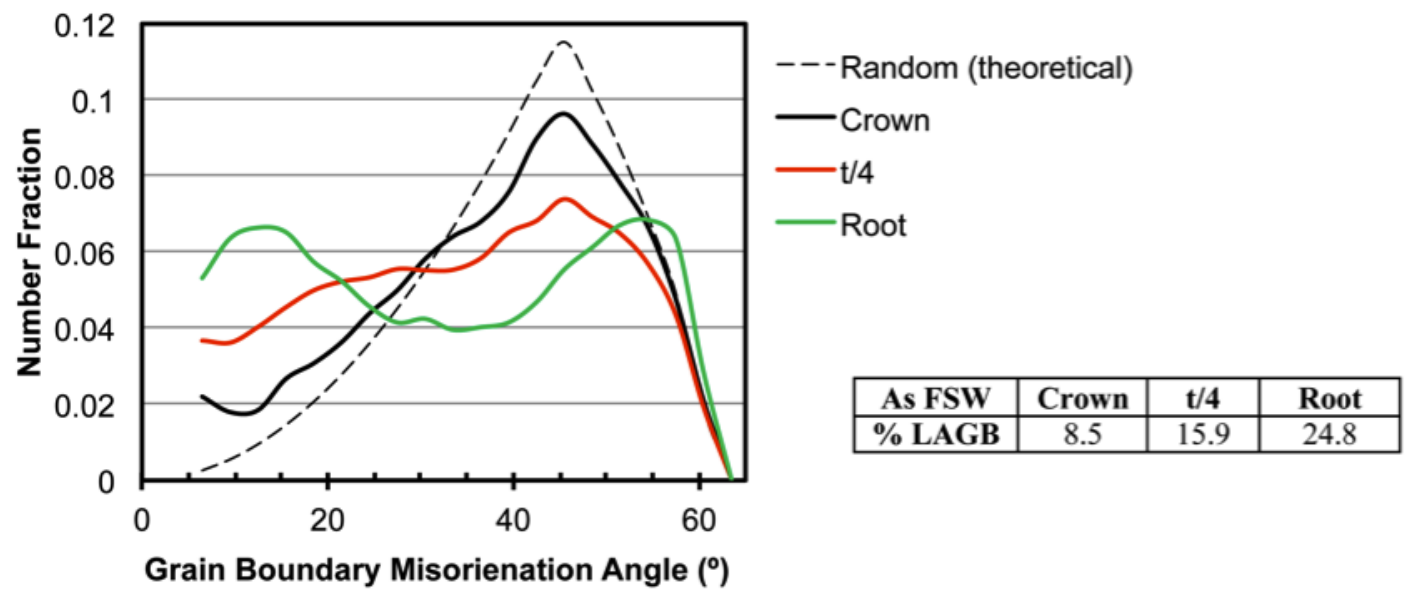

Figure 3 GBMAD and percentage of LAGBs for the As FSW condition.

The ultimate goal of this study is to explore an IAT as a means of stabilizing the microstructure through uniform grain growth prior to SHT to avoid AGG. As evidenced by Figure 1C, the IAT successfully stabilizes the bulk of the weld centered around the $t / 4$ region. Hence, the focus will be directed towards comparing the microstructural variations with processing condition at the $t / 4$ 
region to identify factors that promote AGG in HR condition and factors that suppress AGG after an IAT. This data will then be compared to the spin-formed condition.

In Figure 4, IPF maps for multiple conditions are presented from the $\mathrm{t} / 4$ region of the weld. All conditions have a dominant $<111>$ texture, related to the shear deformation during FSW. PostFSW deformation does not appear significant enough to drastically modify the texture, nor does the IAT. However, there is an appreciable degree of grain growth following the IAT. Average grain sizes are listed in Table 2. In general, the As FSW, After 25\% HR, and As Spin-Formed grain sizes are equivalent with an average grain size of $\sim 7 \mu \mathrm{m}$. After the IAT, there is a $\sim 2 \mathrm{x}$ increase in grain diameter indicating a significant amount of continuous grain growth. However, there are several larger grains in the IPF map for the After IAT condition, revealing that the coarsening was not uniform. For the $\mathrm{t} / 4$ region and bulk of the FSW, the IAT successfully promotes continuous grain growth prior to SHT ( $80 \%$ increase based on average diameter; $\sim 250 \%$ increase based on average area when compared to after $25 \%$ HR condition). Following SHT, the IAT reduces the extent of AGG by $\sim 60 \%$ as compared to the $25 \% \mathrm{HR}+\mathrm{SHT}$ condition (see Figure 1). However, given the variation in AGG after SHT for three conditions (As FSW, After 25\% HR, and As Spin-Formed) that have nominally the same texture and grain size before SHT, it is recognized that additional factors must be considered to explain the varied susceptibility to AGG.
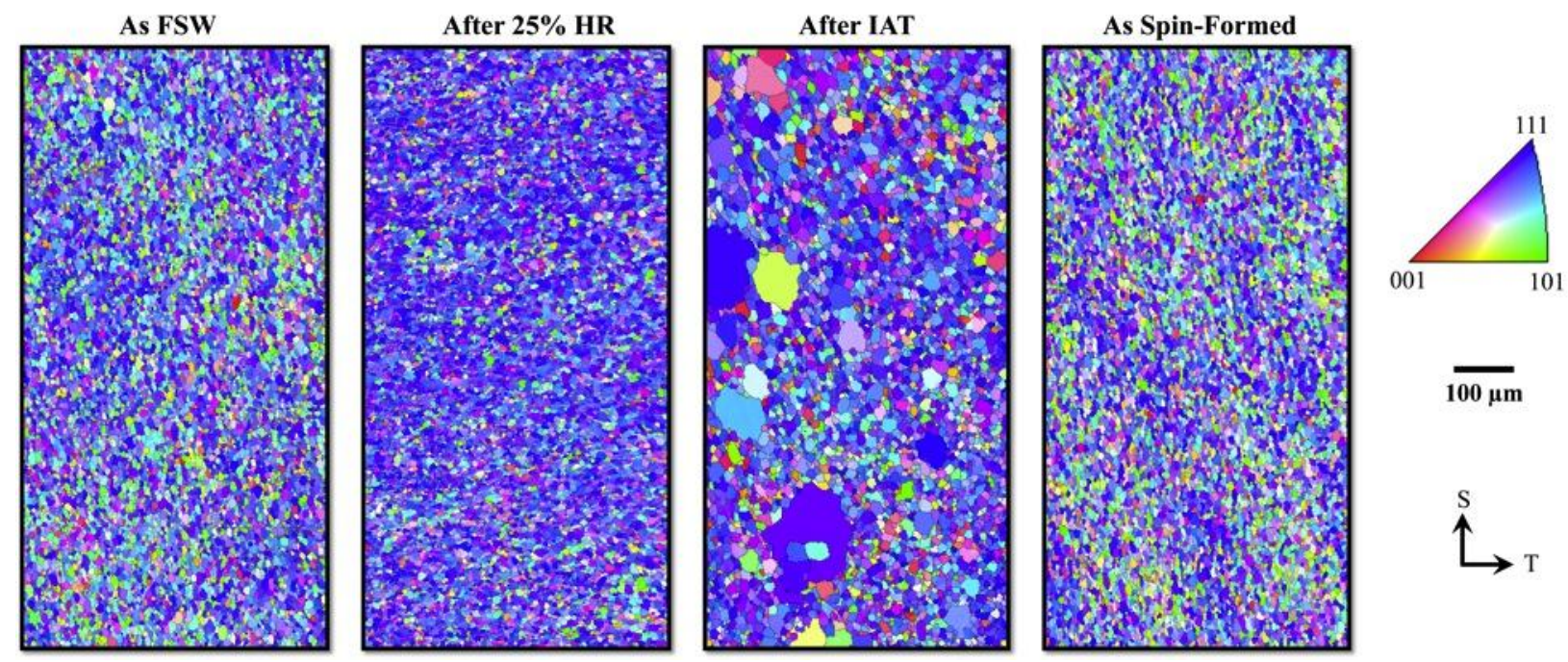

Figure 4 IPF maps from the $t / 4$ region in the conditions indicated above each image.

Table 2 Average grain area in $\mu^{2}$ for each of the conditions indicated at $t / 4$. Average grain diameters in $\mu \mathrm{m}$ are noted in parentheses.

\begin{tabular}{|c|c|c|c|}
\hline As FSW & After 25\% HR & After IAT & As Spin-Formed \\
\hline $41.6(7.3)$ & $32.7(6.5)$ & $112.6(11.9)$ & $42.2(7.3)$ \\
\hline
\end{tabular}

In addition to considering the variations in texture and grain size, it is important to examine the GBMAD and percentage of LAGBs as AGG is related to grain boundary mobility. A plot of the GBMAD for the $\mathrm{t} / 4$ region in various processing conditions is shown in Figure 5, along with the percentage of LAGBs. Of the conditions examined, only the After $25 \%$ HR samples suffered AGG at $\mathrm{t} / 4$. In Figure 5, the After $25 \%$ HR condition stands out from the other samples with a 
higher LAGBs percentage of $\sim 25 \%$. It is reasonable to assume that the rest of the weld would also contain significant percentages of LAGBs, which would explain the massive AGG grains developed throughout the weld region after HR and SHT. The previously documented processing conditions, which did not develop AGG at t/4, were consistently around 15\% LAGBs. An increased percentage of LAGBs indicates a higher level of stored energy (or deformation) within the microstructure, which is a driving force for AGG. Based on the GBMAD, the IAT following HR promotes some level of recovery in addition to grain growth, which serves to lower the stored energy, thereby decreasing the driving force for AGG. Hence, it appears the IAT has a two-fold benefit in that it promotes substantial uniform (continuous) grain growth and recovery leading to a stable grain structure upon SHT at $\mathrm{t} / 4$. In the spin-formed dome, the stored energy due to deformation is minimal in comparison to the HR condition. Thus, the spin-formed material is largely stable upon SHT. The root region at the overlap of the double-pass FSW contains a significant LAGB fraction and has the finest grain size. Hence, this region is the most susceptible to AGG even at PWA temperatures.

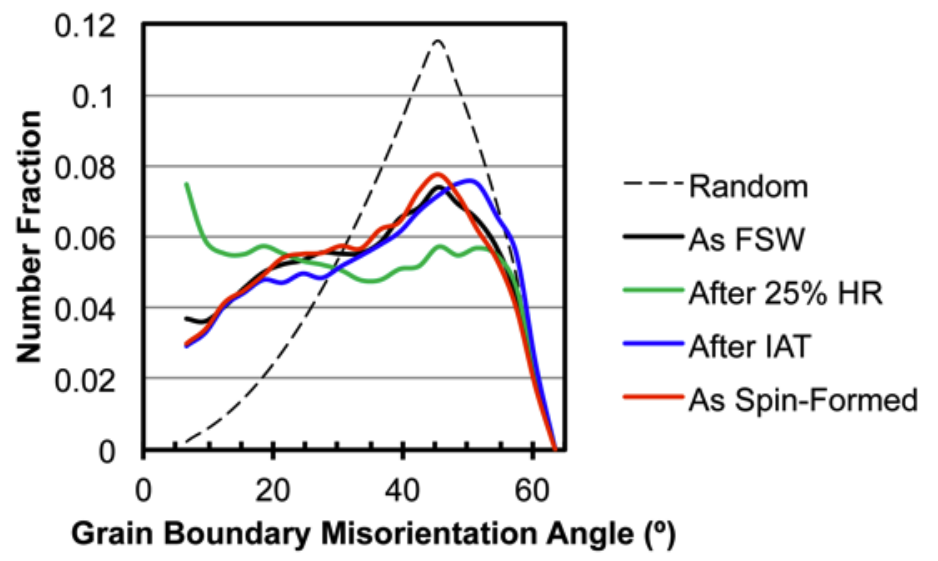

\begin{tabular}{|c|c|}
\hline Condition & \% LAGBs \\
\hline As FSW & 15.9 \\
\hline After 25\% HR & 24.4 \\
\hline After 25\% HR + IAT & 14.7 \\
\hline As Spin-Formed & 15.0 \\
\hline
\end{tabular}

Figure 5 GBMAD and percentage of LAGBs at the $\mathrm{t} / 4$ region for the conditions noted.

\section{Summary}

The near-net shape manufacturing of large-scale launch vehicle structures, like an Orion Crew Module, shows promising reductions in weight and material waste using a combination of FSW and spin-forming. However, forming a plate containing a FSW presents a specific challenge of AGG, which significantly reduces mechanical properties. The current study investigated the occurrence of AGG within the FSW following deformation through hot rolling and spin-forming, as well as an IAT to improve microstructural stability prior to SHT. Several salient findings in relation to AGG have been identified:

- The As FSW microstructure contains grain size, GBMAD, and texture gradients from crown to root. These gradients will influence the magnitude of the driving force for - and the extent of - AGG throughout the weld.

- In the As FSW condition, the region with the highest percentage of LAGBs and smallest grain size is the root. Hence, it is the most susceptible region to AGG.

- The incorporation of an IAT before SHT successfully stabilizes the $25 \% \mathrm{HR}$ microstructure at the $\mathrm{t} / 4$ region by promoting continuous rather than abnormal grain growth and reducing the percentage of LAGBs (stored energy). 
- At the t/4 region, conditions that did not show AGG following SHT had a LAGB percentage of $\sim 15 \%$. Meanwhile, the condition that suffered AGG upon SHT - the HR condition - had approximately $25 \%$ LAGBs. This is similar to a separate study of FSW material that found AGG occurred in cases where the percentage of LAGBs exceeded $18 \%[1]$.

- In comparison to the HR sample, the spin-formed sample had a lower percentage of LAGBs at $\mathrm{t} / 4$. Consequently, the $\mathrm{t} / 4$ region in the spin-formed material does not develop AGG upon SHT. Thus, the increased susceptibility with deformation level may be explained by the resultant increase in LAGBs.

- There remains an unquantified effect of particles and matrix solute content, which may provide a retarding force in response to AGG. Future work should seek to address these issues as they may significantly affect the occurrence of AGG.

\section{References}

1. K. Chen, "The Origin of Coarse Grain Structure in Friction-Stir Welded AA5083 after Heat Treatment" (PhD Thesis, Ohio State University, 2009).

2. I. Charit and R.S. Mishra, "Abnormal grain growth in friction stir processed alloys," Scripta Mater., 58 (5) (2008), 367-371.

3. S.J. Hales and W.A. Tayon, "Heat treatment of a friction-stir-welded and spin-formed Al-Li alloy," Procedia Eng., 10 (0) (2011), 2496-2501.

4. P. Curreri, et al., "Aluminum-Lithium, Friction Stir Welded, Spun-Formed Domes for LightWeight Cryogenic Propellant Tanks Part I: 1-Meter-Diameter Proof of Concept" (NASA/TP-11216462, National Aeronautics and Space Administration, 2011).

5. F.J. Humphreys, "A unified theory of recovery, recrystallization and grain growth, based on the stability and growth of cellular microstructures-I. The basic model," Acta Mater., 45 (10) (1997), 4231-4240.

6. F.J. Humphreys, "A unified theory of recovery, recrystallization and grain growth, based on the stability and growth of cellular microstructures-II. The effect of second-phase particles," Acta Mater., 45 (12) (1997), 5031-5039.

7. R.D. Doherty, et al., "Current issues in recrystallization: a review," Mater. Sci. Eng. A, 238 (2) (1997), 219-274.

8. K.A.A. Hassan, et al., "Stability of nugget zone grain structures in high strength Al-alloy friction stir welds during solution treatment," Acta Mater., 51 (7) (2003), 1923-1936.

9. A.D. Rollett, et al., "Simulation and theory of abnormal grain growth - anisotropic grain boundary energies and mobilities," Acta Metall., 37 (4) (1989), 1227-1240.

10. J.W. Rutter and K.T. Aust, "Migration of $\langle 100\rangle$ tilt grain boundaries in high purity lead," Acta Metall., 13 (3) (1965), 181-186.

11. A.D. Rollett, "Abnormal Grain Growth and Texture Development" (Paper presented at the 14th International Conference on the Texture of Materials (ICOTOM 14), Pittsburgh, PA, 2008).

12. G.S. Crest, et al., "Abnormal Grain Growth in Three Dimensions," Scipte Metallurgica at Materialia, 24 (1990), 661-665.

13. A.D. Rollet, et al, " An Overview of Accomplishments and Challenges in Recrystallization and Grain Growth," Mat. Sci. Forum, 558-559 (2007), 33-42.

14. M.L. Taheri, et al., "In-Situ Quantification of Solute Effects on Grain Boundary Mobility and Character in Aluminum Alloys During Recrystallization," Mat. Sci. Forum, 467-470 (2004),

15. J.K. MacKenzie, "Statistics Associated with the Random Disorientation of Cubes," Biometrika, 45 (229) (1958). 